\title{
A FIXED POINT THEOREM OF THE ALTERNATIVE, FOR CONTRACTIONS ON A GENERALIZED COMPLETE METRIC SPACE
}

\author{
BY J. B. DIAZ AND BEATRIZ MARGOLIS ${ }^{1}$ \\ Communicated by J. B. Diaz, October 18, 1967
}

1. Summary. The purpose of this note is to prove a "theorem of the alternative" for any "contraction mapping" $T$ on a "generalized complete metric space" $X$. The conclusion of the theorem, speaking in general terms, asserts that: either all consecutive pairs of the sequence of successive approximations (starting from an element $x_{0}$ of $X)$ are infinitely far apart, or the sequence of successive approximations, with initial element $x_{0}$, converges to a fixed point of $T$ (what particular fixed point depends, in general, on the initial element $x_{0}$ ). The present theorem contains as special cases both Banach's [1] contraction mapping theorem for complete metric spaces, and Luxemburg's [2] contraction mapping theorem for generalized metric spaces.

2. A fixed point theorem. Following Luxemburg [2, p. 541], the concept of a "generalized complete metric space" may be introduced as in this quotation:

"Let $X$ be an abstract (nonempty) set, the elements of which are denoted by $x, y, \cdots$ and assume that on the Cartesian product $X \times X$ a distance function $d(x, y)(0 \leqq d(x, y) \leqq \infty)$ is defined, satisfying the following conditions

(D1) $d(x, y)=0$ if and only if $x=y$,

(D2) $d(x, y)=d(y, x)$ (symmetry),

(D3) $d(x, y) \leqq d(x, z)+d(z, y)$ (triangle inequality),

(D4) every $d$-Cauchy sequence in $X$ is $d$-convergent, i.e. $\lim _{n, m \rightarrow \infty} d\left(x_{n}, x_{m}\right)=0$ for a sequence $x_{n} \in X(n=1,2, \cdots)$ implies the existence of an element $x \in X$ with $\lim _{n \rightarrow \infty} d\left(x, x_{n}\right)=0,(x$ is unique by (D1) and (D3)).

This concept differs from the usual concept of a complete metric space by the fact that not every two points in $X$ have necessarily a finite distance. One might call such a space a generalized complete metric space."

${ }^{1}$ Research sponsored by Fundación Bariloche, Buenos Aires, Argentina. Now at Universidad Nacional de La Plata, Argentina. 
Using this notion, one has the

THEOREM. Suppose that $(X, d)$ is a generalized complete metric space, and that the function $T: X \rightarrow X$ is a "contraction," that is, $T$ satisfies the condition: (C1) There exists a constant $q$, with $0<q<1$, such that whenever $d(x, y)<\infty$ one has

$$
d(T x, T y) \leqq q d(x, y) .
$$

Let $x_{0} \in X$, and consider the "sequence of successive approximations with initial element $x_{0} ": x_{0}, T x_{0}, T^{2} x_{0}, \cdots, T^{l} x_{0}, \cdots$. Then the following alternative holds: either

(A) for every integer $l=0,1,2, \cdots$, one has

$$
d\left(T^{l} x_{0}, T^{l+1} x_{0}\right)=\infty \text {, or }
$$

(B) the sequence of successive approximations $x_{0}, T x_{0}, T^{2} x_{0}, \cdots$, $T^{l} x_{0}, \cdots$, is $d$-convergent to a fixed point of $T$.

Proof. Consider the sequence of numbers $d\left(x_{0}, T x_{0}\right), d\left(T x_{0}, T^{2} x_{0}\right)$, $\cdots, d\left(T^{l} x_{0}, T^{l+1} x_{0}\right), \cdots$, the "sequence of distances between consecutive neighbors" of the sequence of successive approximations with initial element $x_{0}$. There are two mutually exclusive possibilities: either

(a) for every integer $l=0,1,2, \cdots$, one has

$$
d\left(T^{l} x_{0}, T^{l+1} x_{0}\right)=\infty,
$$

(which is precisely the alternative (A) of the conclusion of the theorem), or else

(b) for some in teger $l=0,1,2, \cdots$, one has

$$
d\left(T^{l} x_{0}, T^{l+1} x_{0}\right)<\infty .
$$

In order to complete the proof it only remains to show that (b) implies alternative $(B)$ of the conclusion of the theorem.

In case (b) holds, let $N=N\left(x_{0}\right)$ denote a particular one (for definiteness, one could choose the smallest) of all the integers $l=0,1,2, \ldots$ such that

$$
d\left(T^{l} x_{0}, T^{l+1} x_{0}\right)<\infty .
$$

Then, by (C1), since $d\left(T^{N} x_{0}, T^{N+1} x_{0}\right)<\infty$, it follows that

$$
d\left(T^{N+1} x_{0}, T^{N+2} x_{0}\right)=d\left(T T^{N} x_{0}, T T^{N+1} x_{0}\right) \leqq q d\left(T^{N} x_{0}, T^{N+1} x_{0}\right)<\infty ;
$$

and, by mathematical induction, that

$$
d\left(T^{N+l} x_{0}, T^{N+l+1} x_{0}\right) \leqq q^{l} d\left(T^{N} x_{0}, T^{N+1} x_{0}\right)<\infty,
$$


for every integer $l=0,1,2, \cdots$. In other words, it has just been proved that, if $n$ is any integer such that $n>N$, then

$$
d\left(T^{n} x_{0}, T^{n+1} x_{0}\right) \leqq q^{n-N} d\left(T^{N} x_{0}, T^{N+1} x_{0}\right)<\infty .
$$

But now, the triangle inequality (D3) implies that, whenever $n>N$, one has, for any $l=1,2, \cdots$, that

$$
\begin{aligned}
d\left(T^{n} x_{0}, T^{n+l} x_{0}\right) & \leqq \sum_{i=1}^{l} d\left(T^{n+i-1} x_{0}, T^{n+i} x_{0}\right) \\
& \leqq \sum_{i=1}^{l} q^{n+i-1-N} d\left(T^{N} x_{0}, T^{N+1} x_{0}\right) \\
& \leqq q^{n-N} \cdot \frac{1-q^{l}}{1-q} \cdot d\left(T^{N} x_{0}, T^{N+1} x_{0}\right)
\end{aligned}
$$

Therefore, since $0<q<1$, the sequence of successive approximations $x_{0}, T x_{0}, T^{2} x_{0}, \cdots, T^{n} x_{0}, \cdots$, is a $d$-Cauchy sequence; and, by (D4), it is $d$-convergent. That is to say, there exists an element $x$ in $X$ such that $\lim _{n \rightarrow \infty} d\left(T^{n} x_{0}, x\right)=0$.

It will now be shown that $x$ is a fixed point of $T$. For, whenever $n>N$, from (D3) and (C1),

$$
\begin{aligned}
0 \leqq d(x, T x) & \leqq d\left(x, T^{n} x_{0}\right)+d\left(T^{n} x_{0}, T x\right) \\
& \leqq d\left(x, T^{n} x_{0}\right)+q d\left(T^{n-1} x_{0}, x\right) ;
\end{aligned}
$$

and thus, taking $\lim _{n \rightarrow \infty}$, it follows that $d(x, T x)=0$. Using (D1), this gives $x=T x$, that is, $x$ is a fixed point of $T$. This completes the proof.

3. Remarks. 1. Banach's [1] contraction mapping theorem is a special case of the present theorem. (Banach's theorem asserts that, if $T$ is a contraction on a complete metric space $X$, then $T$ has exactly one fixed point, and the sequence of successive approximations $x_{0}$, $T x_{0}, T^{2} x_{0}, \cdots, T^{l} x_{0}, \cdots$, for any $x_{0}$ in $X$, always converges to the unique fixed point of $T$.) This can be seen as follows: if $X$ is a complete metric space, then $d(x, y)<\infty$ for every $x, y$ in $X$, and alternative (A) is excluded; since $X$ is not empty, choosing $x_{0} \in X$ gives, from alternative (B), the existence of at least one fixed point of $T$; finally, since $T$ is a contraction, see (C1), $T$ can have at most one fixed point, because if $x=T x$ and $y=T y$ then

$$
d(x, y)=d(T x, T y) \leqq q d(x, y),
$$

which means that $d(x, y)=0$, and $x=y$ from (D1).

2. Luxemburg's [2] contraction mapping theorem for generalized metric spaces is also a special case of the present theorem. (Luxem- 
burg's theorem asserts that, if $T$ is a contraction, i.e. $T$ satisfies (C1), on a generalized complete metric space $X$, and $T$ also satisfies the two additional conditions [2, p. 541]: "(C2) For every sequence of successive approximations $x_{n}=T x_{n-1}, n=1,2, \cdots$, where $x_{0}$ is an arbitrary element of $X$, there exists an index $N\left(x_{0}\right)$ such that $d\left(x_{N}, x_{N+1}\right)<\infty$ for all $l=1,2, \cdots$. (C3) If $x$ and $y$ are two fix points of $T$, i.e. $T x=x$ and $T y=y$, then $d(x, y)<\infty$, then $T$ has exactly one fixed point, and the sequence of successive approximations $x_{0}, T x_{0}, T^{2} x_{0}, \cdots, T^{1} x_{0}, \cdots$, for any $x_{0}$ in $X$, always converges to the unique fixed point of $T$.) This can be seen as follows: in view of hypothesis (C2), alternative (A) is excluded; since $X$ is not empty, choosing $x_{0} \in X$ gives, from alternative (B), the existence of at least one fixed point of $T$; finally, (C3) implies that $T$ can have at most one fixed point, because if $x=T x$ and $y=T y$, with $x \neq y$, then (C3) gives that $d(x, y)<\infty$, while (C1) then yields

$$
d(x, y)=d(T x, T y) \leqq q d(x, y),
$$

which means that $d(x, y)=0$, and $x=y$ from (D1), contradicting the initial $x \neq y$. (This very last bit of reasoning just amounts to saying that if $T$ satisfies (C1), then every two of its fixed points must be infinitely far apart; incidentally, this shows that the situation illustrated in the example in Remark 3 on page 542 of [2] is, in a sense, the rule rather than the exception.)

3. The fixed point theorem of $\$ 2$ applies to a "global" contraction $T$. When $T$ is only "locally" a contraction (see Luxemburg [3, p. 94, condition (C1)]), slight modifications of the proof of $\$ 2$ yield the following "local" theorem of the alternative, which includes Luxemburg's local theorem [3, p. 95] as a special case.

Theorem. Suppose that $(X, d)$ is a generalized complete metric space, and that the function $T: X \rightarrow X$ is locally a contraction, that is, $T$ satisfies the condition

(C1)' There exists a constant $q$, with $0<q<1$, and a positive constant $C$, such that whenever $d(x, y) \leqq C$ one has

$$
d(T x, T y) \leqq q d(x, y) .
$$

Let $x_{0} \in X$, and consider the sequence of successive approximations with initial element $x_{0}: x_{0}, T x_{0}, T^{2} x_{0}, \cdots, T^{l} x_{0}, \cdots$. Then the following alternative holds: either

(A) for every integer $l=0,1,2, \cdots$, one has $d\left(T^{l} x_{0}, T^{l+1} x_{0}\right)>C$, or

(B) the sequence of successive approximations $x_{0}, T x_{0}, T^{2} x_{0}, \cdots$, $T^{l} x_{0}, \cdots$ is $d$-convergent to a fixed point of $T$. 
4. In all the fixed point theorems under discussion, the essential idea is the proof of the convergence of a sequence of successive approximations by means of the geometric series $1+q+q^{2}+\cdots$. This basic idea, of employing this geometric series as a comparison series, goes back to Banach, and to Picard ... .

\section{REFERENCES}

1. S. Banach, Sur les opérations dans les ensembles abstraits et leur application aux équations intégrales (Thèse présentée en juin 1920 a l'Université de Léopol [ $=$ Lwów] pour obtenir le grade de docteur en philosophie), Fund. Math. 3 (1922), 133-181.

2. W. A. J. Luxemburg, On the convergence of successive approximations in the theory of ordinary differential equations. II, Koninkl, Nederl. Akademie van Wetenschappen, Amsterdam, Proc. Ser. A (5) 61, and Indag. Math. (5) 20 (1958), 540-546.

3. W. A. J. Luxemburg, On the convergence of successive approximations in the theory of ordinary differential equations. III, Nieuw Arch. Wisk. (3) 6 (1958), 93-98.

Rensselaer Polytechnic Institute

Universidad Nacional de la Plata, Argentina 